\section{§30. Plasma Plugging in Gas Divertor}

Matsubara, A., Tonegawa, A. (Dept. Phys. Sci. Tokai Univ.)

Murata, R., Ono, M., Sakamoto, S. (Dept Nucl. Eng. Tokai Univ.)

Kawamura, K., Takayama, K. (Res. Inst. Sci. Tech. Tokai Univ.)

Suzuki, H., Watanabe, T., Ohyabu, N., Sato, K.

\section{i) Introduction}

An investigation of a gas divertor in which the high energy ions or electrons collide with low temperature neutral gas atoms to reduce their high energy has originally directed to the formation of detached plasma. The radiated power at the gas divertor also plays an important role in the reduction of the energy of plasma, however, another key issue is how to prevent a reverse flow of gas in the gas divertor toward a main plasma.

As for the plasma pluggings, it was reported that a large pressure difference between the gas divertor and the main plasma could be maintained in a connecting tube, i.e., a narrow cylinder ${ }^{1)}$ or a cylindrical metal tube ${ }^{2)}$. Such cylinders were placed in front of a separating plate between the gas divertor chamber and the plasma source chamber. By contrast with the use of cylinders mentioned above, a separating plate having a tiny hole whose diameter $3 \mathrm{~mm}$ is comparable to the diameter of the string plasma is employed in the present experiment.

\section{ii) Experimental}

The divertor part equipped with both a gas chamber with a small hole of $3 \mathrm{~mm}$ in diameter and a high vacuum chamber is only shown in Fig.1. We expected that such an installation of high-vacuum chamber would prevent the reversed gas flow.

The high purity neon gas $(99.99 \%)$ was used as the divertor gas without further purification and flowed into the divertor. The string plasma drawn out from a plasma source in a String Plasma Device ${ }^{3)}$ by a differential pumping was lead directly in the hole of high-vacuum chamber. The leaked neon gas from the hole of high-vacuum chamber was detected with a quadrupole mass spectrometer (Qmass) at the experimental chamber shown in Fig. 1. The variation of leaked neon was determined as a neon concentration with the different aperture of gate bulbs at both the divertor and the experimental chambers.

iii) Results and Discussions

The variation of leaked neon in the experimental chamber with the discharge-current of plasma is shown in Fig. 2. The leaked neon decreases with increasing the discharge-current of plasma, indicating the appearance of the plasma plugging. In order to provide further evidence that the plasma plugging may exist, the leaked neon is measured as a function of the pressure in high-vacuum chamber at various helium gas flow rate streamed in the plasma source chamber. The obtained relationship between leaked neon and the pressure in high-vacum chamber is shown in Fig. 3. Since the difference of leaked neon between two curves can be considered as the helium plugging due to the change of helium gas flow rate streamed in the plasma source chamber, the effect of plasma plugging on leaked neon may be evaluated qualitatively from two results shown in Figs. 2 and 3. It is found finally that the following equation of the type is proved to be generally adequate to describe the plasma plugging.

$$
\left(P_{1}-P_{2}\right) V=n k\left(T_{1}-T_{2}\right)
$$

where $\mathrm{P}, \mathrm{V}, \mathrm{n}, \mathrm{k}$ and $\mathrm{T}$ are the pressure, the volume, the number of particles and the Boltzmann constant, respectively. The subscript 1 and 2 are referred to a plasma state and no plasma state.

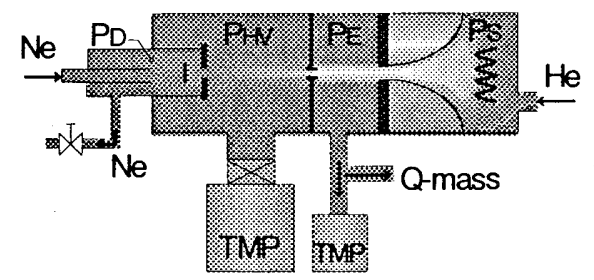

Fig. 1. A Schematic Drawing of Experimental Apparatus. TMP: Turbo Molecular Pump; Q-mass: Quadrupole Mass Spectrometer; $\mathrm{P}_{\mathrm{S}}$ : Pressure in the Plasma Source Chamber; $\mathrm{P}_{\mathrm{E}}$ : Pressure in the Experimental Chamber; $\mathrm{P}_{\mathrm{HV}}$ : Pressure in the High-Vacuum Chamber; $P_{D}$ : Pressure at the Gas Divertor.

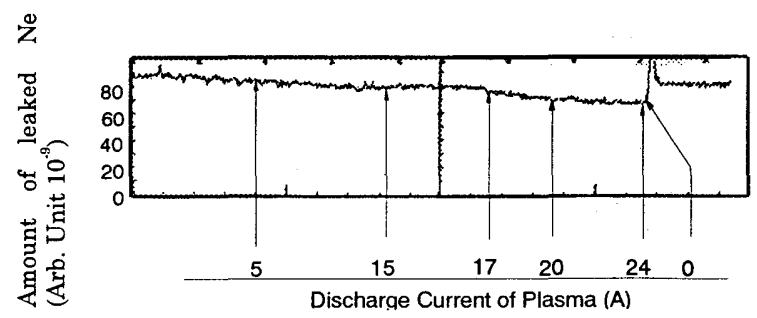

Fig. 2. Variation of the Leaked Neon in the Experimental Chamber with the Discharge Current of Plasma.

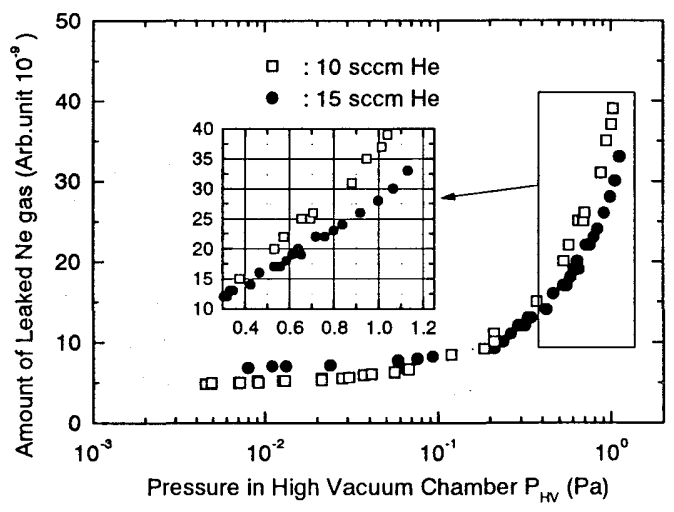

Fig. 3. Relationship between the Leaked Neon in the Experimental chamber and the Presuure in the High Vacuum Chamber.

\section{References}

1) Hsu, W. L. et al. : Bull. Am. Phys. Soc., Ser. 2,24(1979) 992.

2) Hsu, W. L. et al. : Phy. Rev. Letter 49(1982)1001.

3) Tanikawa, T. et al. : Proc. 1989 Int. Conf. on Plasma Physics, (New Delhi 1989), Vol III, p.1157. 